Supporting information

\title{
Engineering of Nitrogen Coordinated Single Cobalt Atom Moieties for Oxygen Electroreduction
}

Wei Sun $^{a}$, Lei Du ${ }^{a}{ }^{*}$, Qiang Tan ${ }^{b}$, Jigang Zhou $^{c}$, Yongfeng Hu ${ }^{c}$, Chunyu Du ${ }^{a}$, Yunzhi Gao ${ }^{a}$, Geping Yin ${ }^{a, *}$

${ }^{a}$ MIIT Key Laboratory of Critical Materials Technology for New Energy Conversion and Storage, Harbin Institute of Technology, Harbin, 150001, China

${ }^{b}$ State Key Laboratory for Mechanical Behavior of Materials, School of Material Science and Engineering, Xi'an Jiaotong University, Xi'an 710049, China

${ }^{c}$ Canadian Light source Inc., Saskatoon, SK S7N 0X4, Canada

Corresponding authors: Dr. Lei Du (lei.du@hit.edu.cn) and Prof. Geping Yin (yingeping@hit.edu.cn) 


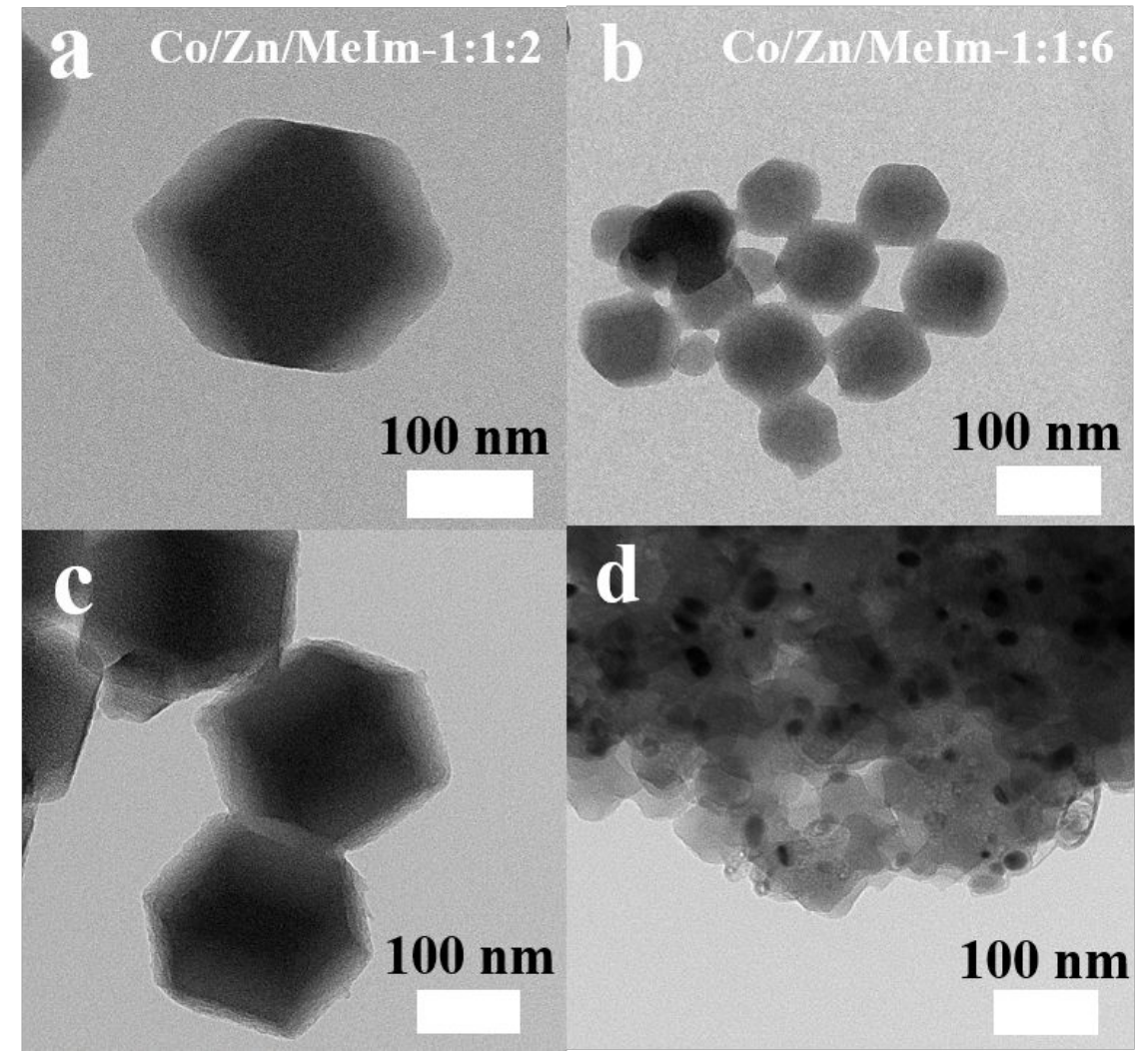

Figure S1. TEM images of ZIF precursors using $\mathrm{Co}\left(\mathrm{NO}_{3}\right)_{2}$ as Co source with $\mathrm{Co} / \mathrm{Zn} / \mathrm{MeIm}$ ratios of (a) 1:1:2, (b) 1:1:6; and (c, d) corresponding TEM images of their derived catalysts after pyrolysis at $900{ }^{\circ} \mathrm{C}$ for $3 \mathrm{~h}$. 

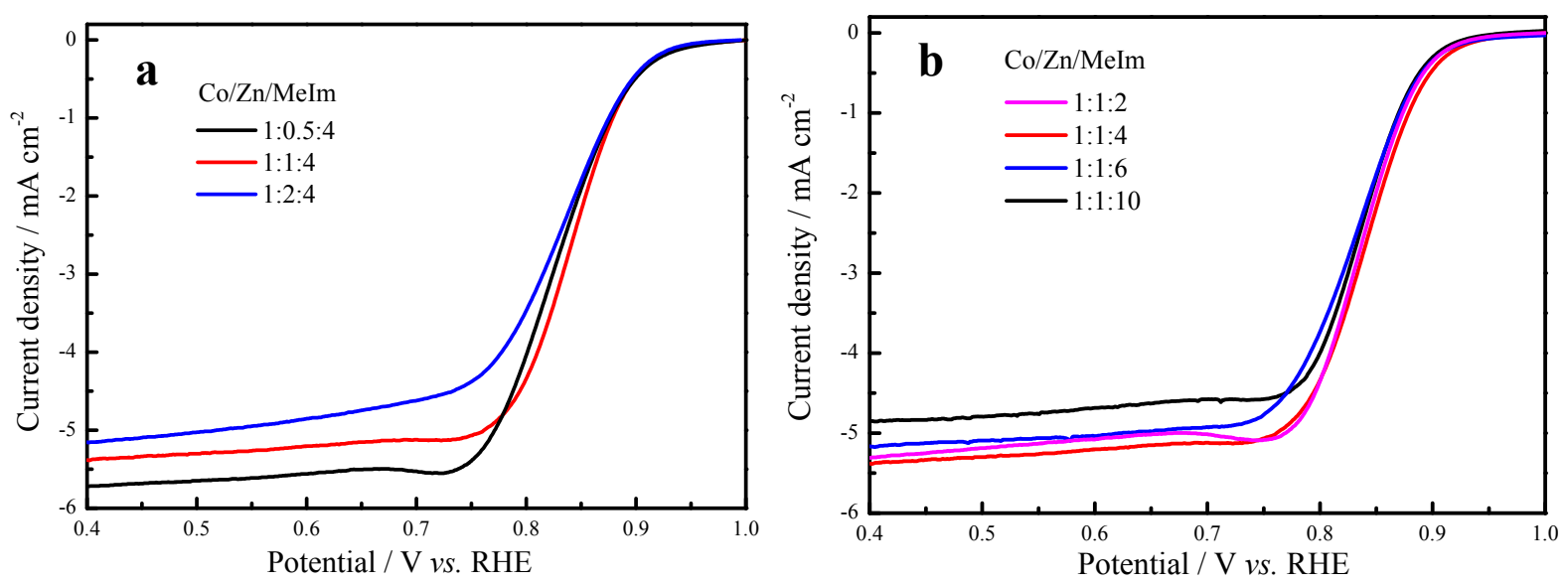

Figure S2. LSV curves of derived catalysts using different Co/Zn/MeIm ratios: the changed (a) zinc and (b) MeIm contents. 


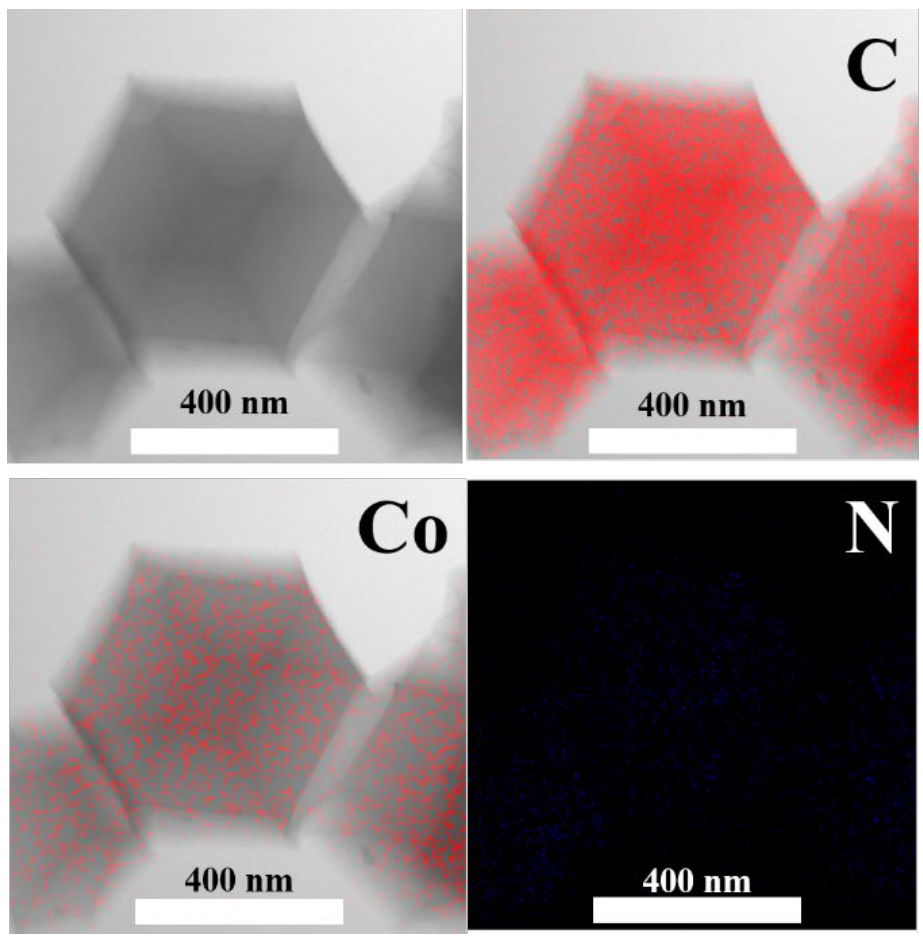

Figure S3. HRTEM image and element mapping of Co/N/C-1:1:4. 


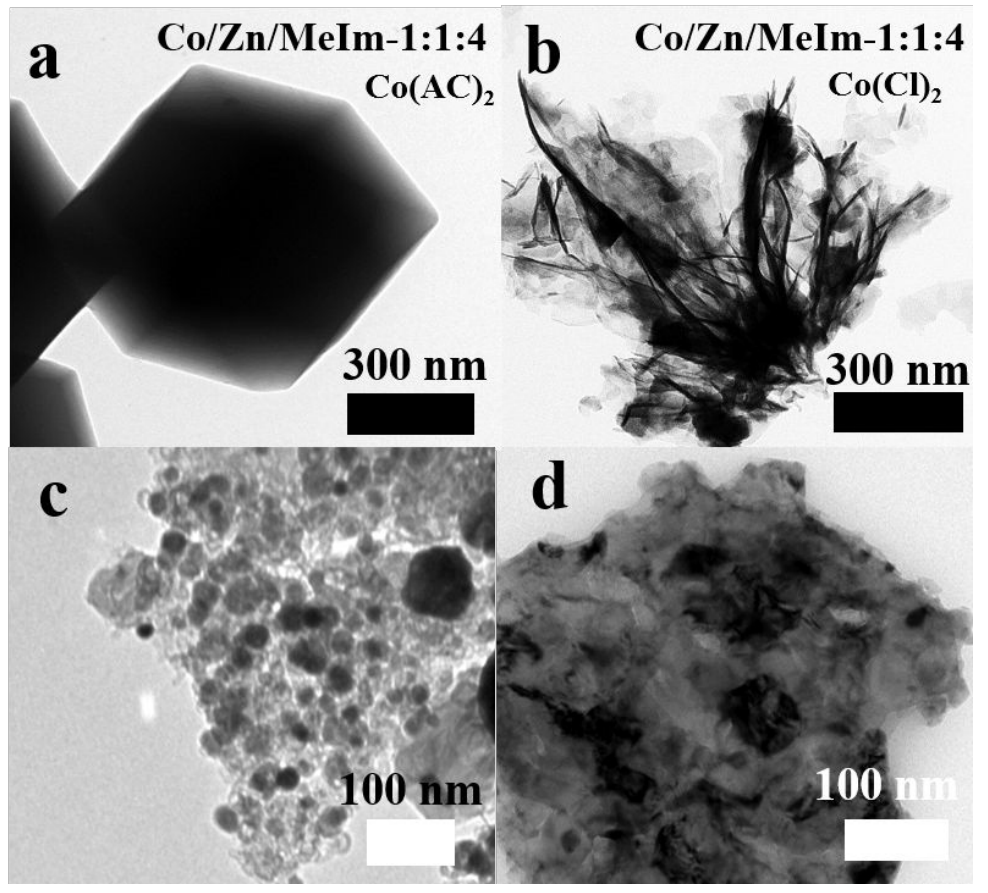

Figure S4. TEM images of Co-doped ZIF precursors using (a) $\mathrm{Co}(\mathrm{AC})_{2}$ and (b) $\mathrm{CoCl}_{2}$ as Co source with $\mathrm{Co} / \mathrm{Zn} / \mathrm{MeIm}=1: 1: 4$, and $(\mathrm{c}, \mathrm{d})$ corresponding TEM images of their derived catalysts after pyrolysis at $900{ }^{\circ} \mathrm{C}$ for $3 \mathrm{~h}$. 


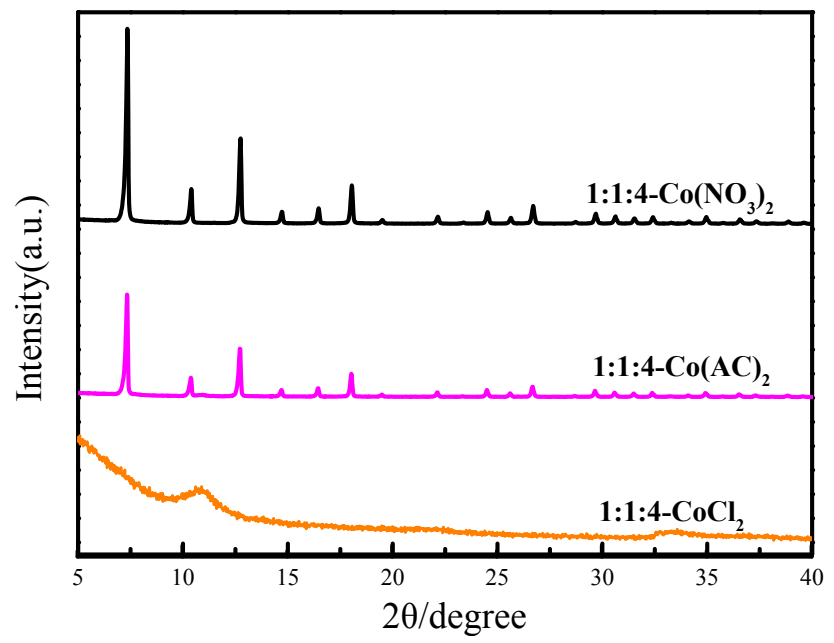

Figure S5. XRD patterns of ZIF precursors using different cobalt sources. 


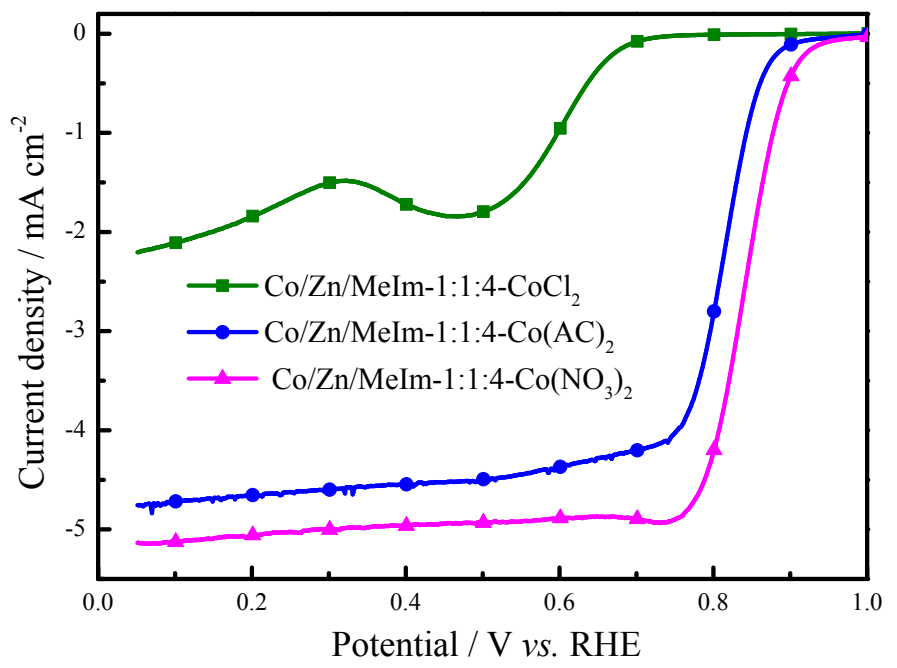

Figure S6. LSV curves of Co/N/C catalysts using different cobalt source with $\mathrm{Co} / \mathrm{Zn} / \mathrm{MeIm}=1: 1: 4$ atomic ratio. 


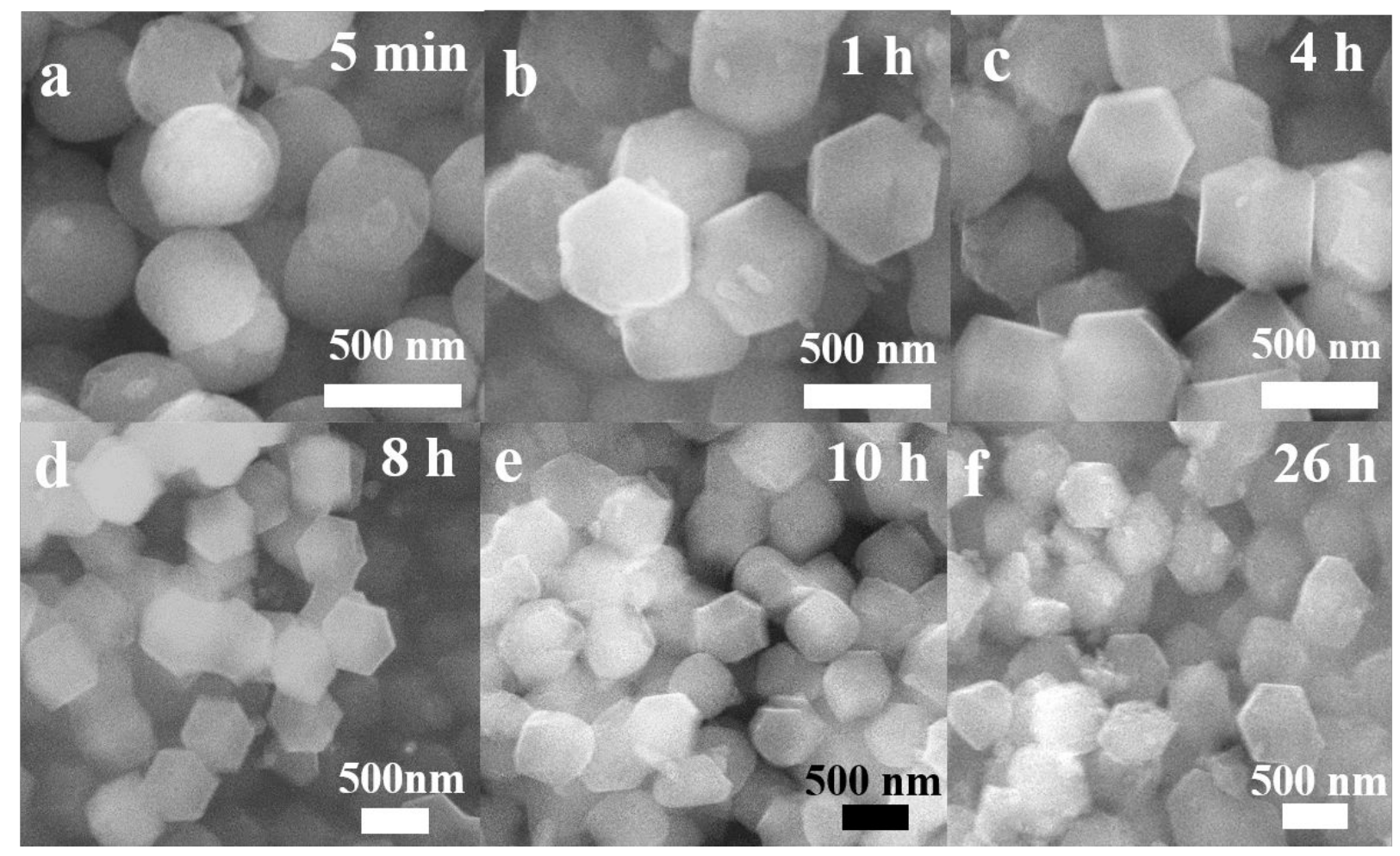

Figure S7. SEM images of synthesized ZIF precursors using $\mathrm{Co}\left(\mathrm{NO}_{3}\right)_{2}$ as Co source with $\mathrm{Co} / \mathrm{Zn} / \mathrm{MeIm}$ ratio of 1:1:4 after reacting for (a) $5 \mathrm{~min}$, (b) $1 \mathrm{~h}$, (c) $4 \mathrm{~h}$, (d) $8 \mathrm{~h},(\mathrm{e}) 10 \mathrm{~h}$, and (f) $26 \mathrm{~h}$. 


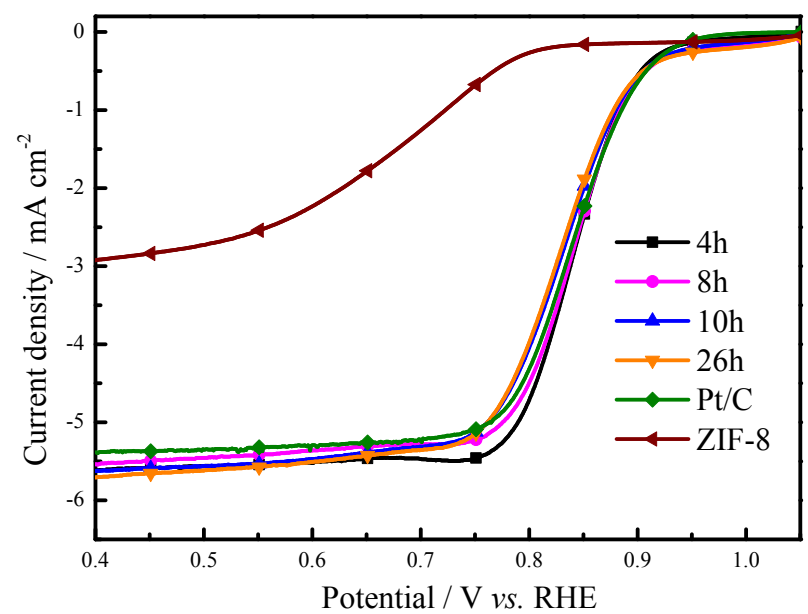

Figure S8. LSV curves of catalysts derived from Co-doped ZIF after different reaction time. 

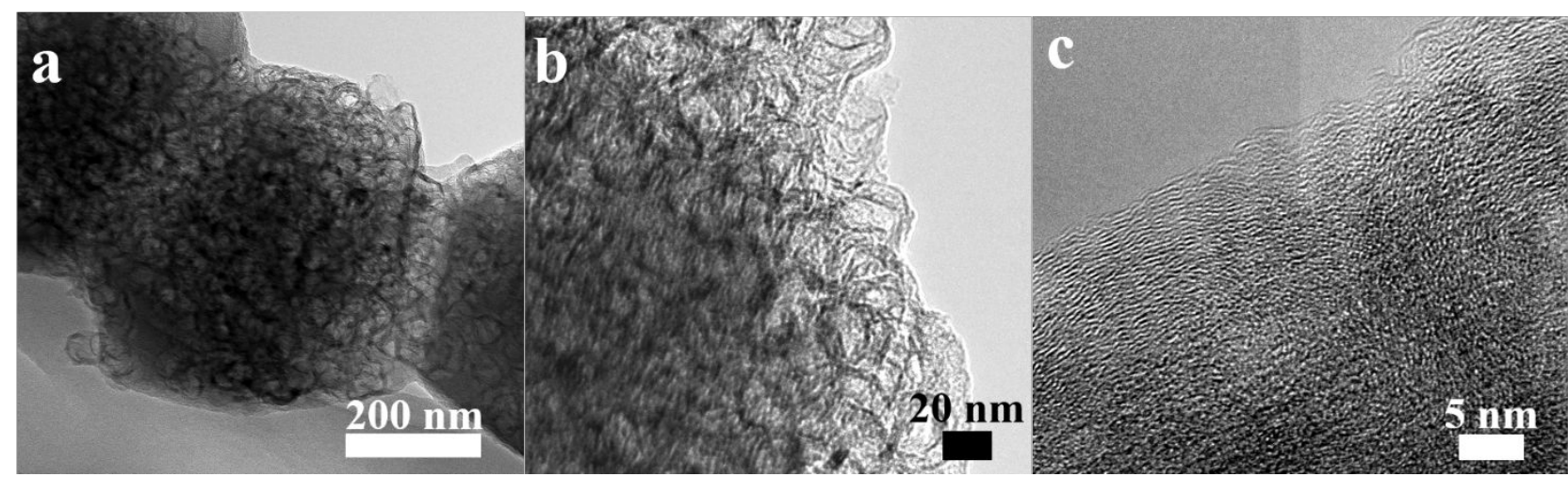

Figure S9. (a) The TEM image and (b) HRTEM image of Co/N/C-1100; (c) the HRTEM image of graphitized carbon in $\mathrm{Co} / \mathrm{N} / \mathrm{C}-1100$ catalyst. 

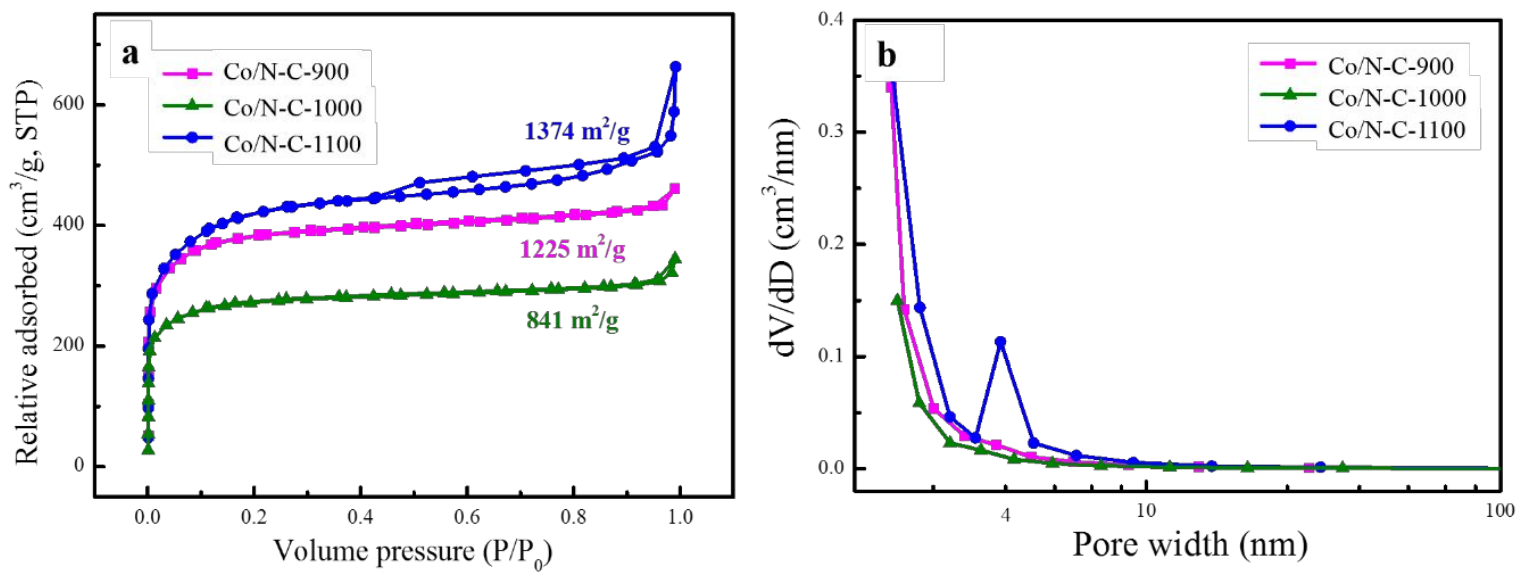

Figure S10. (a) The nitrogen adsorption/desorption isotherms and (b) pore size distribution of $\mathrm{Co} / \mathrm{N} / \mathrm{C}$ catalysts using different pyrolysis temperatures.

As the pyrolysis temperature increasing from $900{ }^{\circ} \mathrm{C}$ to $1000{ }^{\circ} \mathrm{C}$, the surface area somehow decreases, the reason of which is under investigation. By applying $1100{ }^{\circ} \mathrm{C}$, the highest surface area is obtained likely due to the almost complete evaporation of $\mathrm{Zn}$; while mesopores are obtained, likely due to the collapse of micropores. 


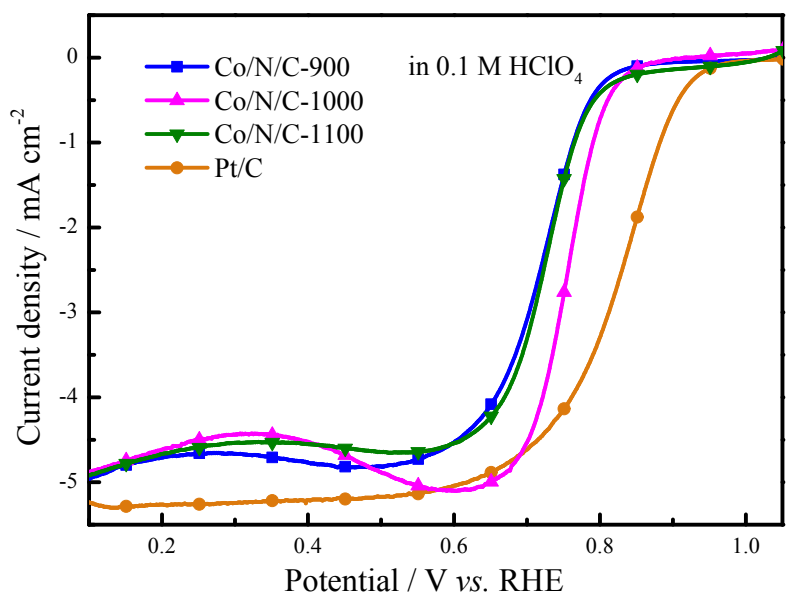

Figure S11. LSV curves of $\mathrm{Co} / \mathrm{N} / \mathrm{C}$ catalysts at different pyrolysis temperature compared with $\mathrm{Pt} / \mathrm{C}$ in $0.1 \mathrm{M} \mathrm{HClO}_{4}$ solutions. 


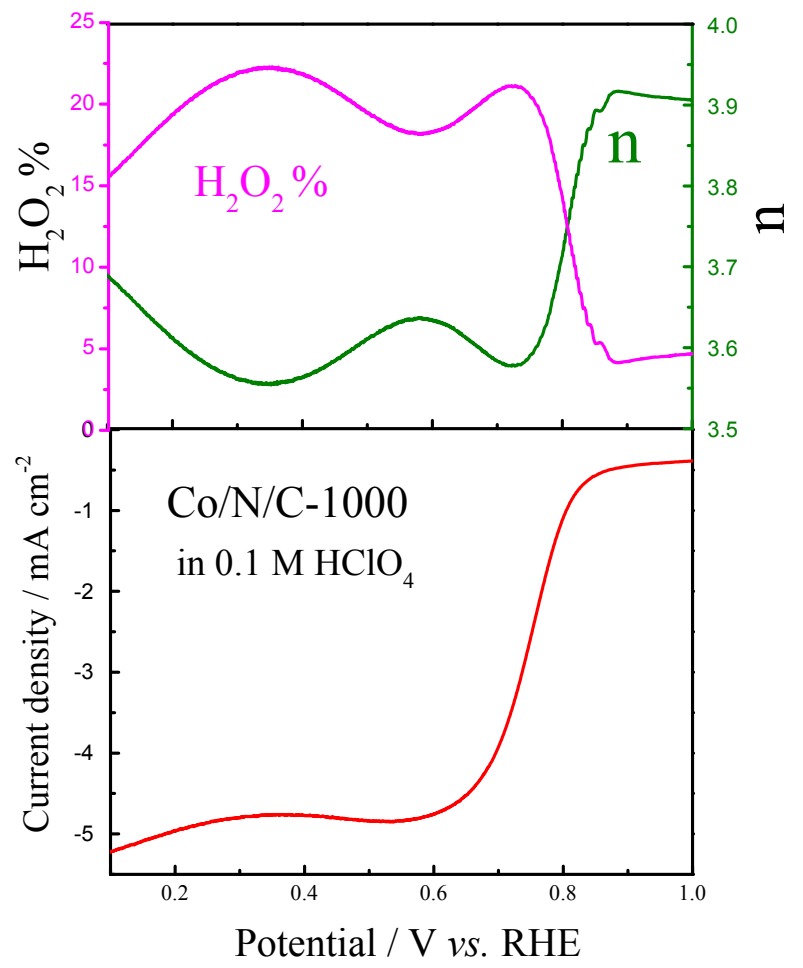

Figure S12. Electron transfer number (n; green) and $\mathrm{H}_{2} \mathrm{O}_{2}$ yield (pink) of $\mathrm{Co} / \mathrm{N} / \mathrm{C}-1000$ catalyst in $0.1 \mathrm{M} \mathrm{HClO}_{4}$ solution. 
Table S1. Compared half-wave potential $\left(\mathrm{E}_{1 / 2}\right)$ and kinetic current density at $0.9 \mathrm{~V}$ of the derived $\mathrm{Co} / \mathrm{N} / \mathrm{C}$ catalysts using different $\mathrm{Co} / \mathrm{Zn} / \mathrm{MeIm}$ ratios at different pyrolysis temperatures.

\begin{tabular}{|c|c|c|}
\hline Derived Catalysts & $\mathrm{E}_{1 / 2}(\mathrm{~V})$ & $\mathrm{I}_{\mathrm{k}} @ 0.9 \mathrm{~V}\left(\mathrm{~mA} \mathrm{~cm}^{-2}\right)$ \\
\hline $1: 0.5: 4-900$ & 0.831 & 0.484 \\
\hline $1: 2: 4-900$ & 0.837 & 0.532 \\
\hline $1: 1: 2-900$ & 0.84 & 0.356 \\
\hline $1: 1: 6-900$ & 0.836 & 0.345 \\
\hline $1: 1: 10-900$ & 0.831 & 0.303 \\
\hline $1: 1: 4-900$ & 0.843 & 0.442 \\
\hline $1: 1: 4-1000$ & 0.856 & 0.641 \\
\hline $1: 1: 4-1100$ & 0.853 & 0.421 \\
\hline $\mathrm{Pt} / \mathrm{C}$ & 0.84 & 0.645 \\
\hline
\end{tabular}


Table S2. ICP results of atomic $\mathrm{Co} \%$ of $\mathrm{Co} / \mathrm{N} / \mathrm{C}$ catalysts with different $\mathrm{ZIF}$ reaction time.

\begin{tabular}{|c|c|}
\hline reaction time & Co (at. \%) \\
\hline $4 \mathrm{~h}$ & $1.77 \%$ \\
\hline $8 \mathrm{~h}$ & $2 \%$ \\
\hline $26 \mathrm{~h}$ & $1.05 \%$ \\
\hline
\end{tabular}


Table S3. Detailed parameters of deconvoluted N 1s XPS spectra.

\begin{tabular}{|c|c|c|c|c|c|c|c|c|c|c|}
\hline \multirow{2}{*}{ Catalysts } & \multicolumn{2}{|c|}{ Pyridinic-N } & \multicolumn{2}{|c|}{ Pyrrolic-N } & \multicolumn{2}{|c|}{$\mathrm{Co}-\mathrm{N}_{\mathrm{x}}$} & \multicolumn{2}{|c|}{ graphite-N } & \multicolumn{2}{|c|}{ Oxide-N } \\
\hline & Position & content & Position & content & Position & content & Position & content & Position & content \\
\hline $\mathrm{Co} / \mathrm{N} / \mathrm{C}-900$ & $398.1 \mathrm{eV}$ & $15.4 \%$ & $399.8 \mathrm{eV}$ & $17.7 \%$ & $398.7 \mathrm{eV}$ & $32.3 \%$ & $401.2 \mathrm{eV}$ & $34.6 \%$ & \multicolumn{2}{|c|}{0} \\
\hline $\mathrm{Co} / \mathrm{N} / \mathrm{C}-1000$ & $398.1 \mathrm{eV}$ & $19.5 \%$ & $399.9 \mathrm{eV}$ & $16.6 \%$ & $398.7 \mathrm{eV}$ & $38.8 \%$ & $401.06 \mathrm{eV}$ & $25.1 \%$ & \multicolumn{2}{|c|}{0} \\
\hline $\mathrm{Co} / \mathrm{N} / \mathrm{C}-1100$ & $398.2 \mathrm{eV}$ & $9.6 \%$ & $399.9 \mathrm{eV}$ & $5.9 \%$ & $399 \mathrm{eV}$ & $22.3 \%$ & $401.08 \mathrm{eV}$ & $49 \%$ & $403.2 \mathrm{eV}$ & $13.2 \%$ \\
\hline
\end{tabular}


Table S4. The XPS atomic contents of $\mathrm{Zn}, \mathrm{Co}$ and $\mathrm{N}$ in $\mathrm{Co} / \mathrm{N} / \mathrm{C}$ catalysts at different pyrolysis temperatures.

\begin{tabular}{|c|c|c|c|}
\hline Catalysts & Zn (at. \%) & Co (at. \%) & N (at. \%) \\
\hline Co/N/C-900 & 2.31 & 0.75 & 10.65 \\
\hline Co/N/C-1000 & 1.89 & 1.12 & 8.14 \\
\hline Co/N/C-1100 & 0 & 0.62 & 3.3 \\
\hline
\end{tabular}


Table S5. ICP results of atomic $\mathrm{Co} \%$ and $\mathrm{Zn} \%$ of $\mathrm{Co} / \mathrm{N} / \mathrm{C}$ catalysts at different pyrolysis temperatures.

\begin{tabular}{|c|c|c|}
\hline Catalysts & Zn (at. \%) & Co (at. \%) \\
\hline Co/N/C-900 & 4.02 & 2 \\
\hline Co/N/C-1000 & 2.33 & 3.02 \\
\hline Co/N/C-1100 & 0 & 1.88 \\
\hline
\end{tabular}


Table S6. Compared alkaline and acidic ORR activity between Co-N-C-1000 catalyst in this work and other typical nonprecious catalysts in literature.

\begin{tabular}{|c|c|c|c|c|}
\hline Electrocatalysts & $\mathrm{E}_{1 / 2}(\mathrm{~V}$ vs RHE) & & Structures & Reference \\
\hline \multirow{2}{*}{ Co-N-C-1000 } & $0.856 \mathrm{~V}$ & $0.1 \mathrm{M} \mathrm{KOH}$ & \multirow{3}{*}{ Co atmos } & \multirow{2}{*}{ This work } \\
\hline & $075 \mathrm{~V}$ & $01 \mathrm{MHClO}$ & & \\
\hline & & & & \\
\hline \multirow{2}{*}{$\mathrm{Co}-\mathrm{N}-\mathrm{C}$} & $0.9 \mathrm{~V}$ & $0.1 \mathrm{M} \mathrm{KOH}$ & \multirow{2}{*}{ Co nanoparticles } & \multirow{2}{*}{1} \\
\hline & $0.5 \mathrm{~V}$ & $0.1 \mathrm{M} \mathrm{HClO}_{4}$ & & \\
\hline C-95Zn5Co & $0.89 \mathrm{~V}$ & $0.1 \mathrm{M} \mathrm{KOH}$ & Co nanoparticles & 2 \\
\hline $\mathrm{CuCo@NC}$ & $0.884 \mathrm{~V}$ & $0.1 \mathrm{M} \mathrm{KOH}$ & Co nanoparticles & 3 \\
\hline Co SAs/N-C(900) & $0.881 \mathrm{~V}$ & $0.1 \mathrm{M} \mathrm{KOH}$ & Co atmos & 4 \\
\hline $\mathrm{Co} / \mathrm{NC}$ hollow sphere & $0.87 \mathrm{~V}$ & $0.1 \mathrm{M} \mathrm{KOH}$ & Co nanoparticles & 5 \\
\hline \multirow[b]{2}{*}{$\mathrm{Co}-\mathrm{N}-\mathrm{C}$} & $0.87 \mathrm{~V}$ & $0.1 \mathrm{M} \mathrm{KOH}$ & \multirow[b]{2}{*}{ Co nanoparticles } & \multirow{2}{*}{6} \\
\hline & $0.761 \mathrm{~V}$ & $0.1 \mathrm{M} \mathrm{HClO}_{4}$ & & \\
\hline Nano-P-ZIF-67 & $0.86 \mathrm{~V}$ & $0.1 \mathrm{M} \mathrm{KOH}$ & Co nanoparticles & 7 \\
\hline Co,N-HCNP & $0.855 \mathrm{~V}$ & $0.1 \mathrm{M} \mathrm{KOH}$ & Co nanoparticles & 8 \\
\hline CoZn-x & $0.85 \mathrm{~V}$ & $0.1 \mathrm{M} \mathrm{KOH}$ & Co nanoparticles & 9 \\
\hline $\mathrm{CoNx}$ & $0.846 \mathrm{~V}$ & $0.1 \mathrm{M} \mathrm{KOH}$ & single clusters & 10 \\
\hline \multirow{2}{*}{$\mathrm{Co}-\mathrm{N} / \mathrm{C}$} & $0.84 \mathrm{~V}$ & $0.1 \mathrm{M} \mathrm{KOH}$ & \multirow{2}{*}{$\begin{array}{l}\text { Co and } \mathrm{Co}_{9} \mathrm{~S}_{8} \\
\text { nanoparticles }\end{array}$} & \multirow{2}{*}{11} \\
\hline & $0.5 \mathrm{~V}$ & $0.1 \mathrm{M} \mathrm{HClO}_{4}$ & & \\
\hline \multirow{2}{*}{ ZIF-67-900 } & $0.82 \mathrm{~V}$ & $0.1 \mathrm{M} \mathrm{KOH}$ & \multirow{2}{*}{ Co nanoparticles } & \multirow{2}{*}{12} \\
\hline & $0.75 \mathrm{~V}$ & $0.1 \mathrm{M} \mathrm{HClO}_{4}$ & & \\
\hline \multirow[b]{2}{*}{ Co- $\mathrm{N}_{4}$} & $0.825 \mathrm{~V}$ & $0.1 \mathrm{M} \mathrm{KOH}$ & \multirow[b]{2}{*}{ Co atmos } & \multirow[b]{2}{*}{13} \\
\hline & $0.8 \mathrm{~V}$ & $0.1 \mathrm{M} \mathrm{H}_{2} \mathrm{SO}_{4}$ & & \\
\hline Co-P,N-CNT & $0.8 \mathrm{~V}$ & $0.1 \mathrm{M} \mathrm{KOH}$ & Co atmos & 14 \\
\hline \multirow{2}{*}{$\mathrm{Co}-\mathrm{N} / \mathrm{CNFs}$} & $0.82 \mathrm{~V}$ & $0.1 \mathrm{M} \mathrm{KOH}$ & \multirow[b]{2}{*}{ Co atmos } & \multirow[b]{2}{*}{15} \\
\hline & $0.7 \mathrm{~V}$ & $0.1 \mathrm{M} \mathrm{HClO}_{4}$ & & \\
\hline Co-N-C-NS & $0.82 \mathrm{~V}$ & $0.1 \mathrm{M} \mathrm{KOH}$ & Co nanoparticles & 16 \\
\hline Co@NC & $0.79 \mathrm{~V}$ & $0.1 \mathrm{M} \mathrm{KOH}$ & Co nanoparticles & 17 \\
\hline \multirow{2}{*}{$\mathrm{FeCo}-\mathrm{NC}$} & $0.83 \mathrm{~V}$ & $0.1 \mathrm{M} \mathrm{KOH}$ & \multirow{2}{*}{ Atomic metal doping } & \multirow{2}{*}{18} \\
\hline & $0.74 \mathrm{~V}$ & $0.1 \mathrm{M} \mathrm{HClO}_{4}$ & & \\
\hline \multirow[b]{2}{*}{ Co-N-C-900 } & $0.8 \mathrm{~V}$ & $0.1 \mathrm{M} \mathrm{KOH}$ & \multirow[b]{2}{*}{ Co nanoparticles } & \multirow[b]{2}{*}{19} \\
\hline & $0.5 \mathrm{~V}$ & $0.1 \mathrm{M} \mathrm{HClO}_{4}$ & & \\
\hline
\end{tabular}


References:

(1) Palaniappan, S.; Roopathy, M.; Alex, Schechter. Unraveling the Oxygen-Reduction Sites in GraphiticCarbon Co-N-C-Type Electrocatalysts Prepared by SinglePrecursor Pyrolysis. ChemCatChem 2017, 9, 1969-1978.

(2) Wang, X.; Fan, X.; Lin, H.; Fu, H.; Wang, T.; Zheng, J.; Li, X. An Efficient Co-N-C Oxygen Reduction Catalyst with Highly Dispersed Co Sites Derived from A ZnCo Bimetallic Zeolitic Imidazolate Framework. Rsc Advances 2016, 6, 37965-37973.

(3) Kuang, M.; Wang, Q.; Han, P.; Zheng, G. Cu, Co-Embedded N-Enriched Mesoporous Carbon for Efficient Oxygen Reduction and Hydrogen Evolution Reactions. Advanced Energy Materials 2017, 7, 1700193-1700200.

(4) Yin, P.; Yao, T.; Wu, Y.; Zheng, L.; Lin, Y.; Liu, W.; Ju, H.; Zhu, J.; Hong, X.; Deng, Z.; Zhou, G.; Wei, S.; Li, Y. Single Cobalt Atoms with Precise N-Coordination as Superior Oxygen Reduction Reaction Catalysts. Angew Chem Int Edit 2016, 55, 10800-10805.

(5) Guan, B.; Yu, L.; Lou, X. Formation of Single-Holed Cobalt/N-Doped Carbon Hollow Particles with Enhanced Electrocatalytic Activity toward Oxygen Reduction Reaction in Alkaline Media. Advanced Science 2017, 4, 1700247-1700252.

(6) You, B.; Jiang, N.; Sheng, M.; Drisdell, W. S.; Yano, J.; Sun, Y. Bimetal-Organic Framework Self-Adjusted Synthesis of Support-Free Nonprecious Electrocatalysts for Efficient Oxygen Reduction. ACS Catalysis 2015, 5, 7068-7076.

(7) Ai, K.; Li, Z.; Cui, X. Scalable Preparation of Sized-Controlled Co-N-C Electrocatalyst for Efficient Oxygen Reduction Reaction. Journal of Power Sources 2017, 368, 46-56.

(8) Hu, Z.; Zhang, Z.; Li, Z.; Dou, M.; Wang, F. One-Step Conversion from Core-Shell MetalOrganic Framework Materials to Cobalt and Nitrogen Codoped Carbon Nanopolyhedra with Hierarchically Porous Structure for Highly Efficient Oxygen Reduction. ACS Applied Materials \& Interfaces 2017, 9, 16109-16116.

(9) Li, Z.; Shao, M.; Zhou, L.; Yang, Q.; Zhang, C.; Wei, M.; Evans, D. G.; Duan, X. Carbon-Based Electrocatalyst Derived from Bimetallic Metal-Organic Framework Arrays for High Performance Oxygen Reduction. Nano Energy 2016, 25, 100-109.

(10) He, T.; Xue, H.; Wang, X.; He, S.; Lei, Y.; Zhang, Y.; Shen, R.; Zhang, Y.; Xiang, J. Architecture of CoNx Single Clusters on Nanocarbon as Excellent Oxygen Reduction Catalysts with HighEfficient Atomic Utilization. Nanoscale 2017, 9, 8341-8348.

(11) Wang, J.; Li, L.; Chen, X.; Lu, Y.; Yang, W.; Duan, X. A Co-N/C Hollow-sphere Electrocatalyst Derived from a Metanilic CoAl Layered Double Hydroxide for the Oxygen Reduction Reaction, and Its Active Sites in Various pH Media. Nano Research 2017, 10, 2508-2518.

(12) Wang, X.; Zhou, J.; Fu, H.; Li, W.; Fan, X.; Xin, G.; Zheng, J.; Li, X. MOF Derived Catalysts for Electrochemical Oxygen Reduction. Journal of Materials Chemistry A 2014, 2, 14064-14070.

(13) Wang, X.; Cullen, D.; Pan, Y.; Hwang, S.; Wang, M.; Feng, Z.; Wang, J.; Engelhard, M. H.; Zhang, H.; He, Y.; Shao, Y.; Su, D.; More, K. L.; Spendelow, J. S.; Wu, G. NitrogenCoordinated Single Cobalt Atom Catalysts for Oxygen Reduction in Proton Exchange Membrane Fuel Cells. Advanced Materials 2018, 30, 1706758-1706768.

(14) Guo, S.; Yuan, P.; Zhang, J.; Jin, P.; Sun, H.; Lei, K.; Pang, X.; Xu, Q.; Cheng, F. Atomic-Scaled Cobalt Encapsulated in P, N-doped Carbon Sheaths over Carbon Nanotubes for Enhanced Oxygen Reduction Electrocatalysis under Acidic and Alkaline Media. Chemical Communications 2017, 53, 9862-9865.

(15) Cheng, Q.; Yang, L.; Zou, L.; Zou, Z.; Chen, C.; Hu, Z.; Yang, H. Single Cobalt Atom and N Codoped Carbon Nanofibers as Highly Durable Electrocatalyst for Oxygen Reduction Reaction. ACS Catalysis 2017, 7, 6864-6871.

(16) Lee, J.; Park, M.; Yoo, S.; Jang, J.; Kim, H.; Nam, S.; Yoon, C.; Kim, J. A Highly Active and 
Durable Co-N-C Electrocatalyst Synthesized Using Exfoliated Graphitic Carbon Nitride Nanosheets. Nanoscale 2015, 7, 10334-10339.

(17) Li, X.; Jiang, Q.; Dou, S.; Deng, L.; Huo, J.; Wang, S. ZIF-67-Eerived Co-NC@CoP-NC Nanopolyhedra as an Efficient Bifunctional Oxygen Electrocatalyst. Journal of Materials Chemistry A 2016, 4, 15836-15840.

(18) Zhang, Z.; Dou, M.; Liu, H.; Dai, L.; Wang, F. A Facile Route to Bimetal and Nitrogen-Codoped 3D Porous Graphitic Carbon Networks for Efficient Oxygen Reduction. Small 2016, 12 (31), 4193-4199.

(19) Guo, C.; Wu, Y.; Li, Z.; Liao, W.; Sun, L.; Wang, C.; Wen, B.; Li, Y.; Chen, C. The Oxygen Reduction Electrocatalytic Activity of Cobalt and Nitrogen Co-doped Carbon Nanocatalyst Synthesized by a Flat Template. Nanoscale Research Letters 2017, 12, 1706-1712. 